Journal Club

Editor's Note: These short reviews of recent JNeurosci articles, written exclusively by students or postdoctoral fellows, summarize the important findings of the paper and provide additional insight and commentary. If the authors of the highlighted article have written a response to the Journal Club, the response can be found by viewing the Journal Club at www.jneurosci.org. For more information on the format, review process, and purpose of Journal Club articles, please see http://jneurosci.org/content/ preparing-manuscript\#journalclub.

\title{
Understanding the Hierarchical Organization of Large-Scale Networks Based on Temporal Modulations in Patterns of Neural Connectivity
}

\author{
Reema Shafi \\ Rehabilitation Sciences Institute, University of Toronto, Toronto, Ontario M5G 1V7, Canada \\ Review of Shine et al.
}

Marsel Mesulam's (Mesulam, 1990) vision that "complex behavior is mapped at the level of multifocal neural systems rather than specific anatomical sites" has triggered an overwhelming interest in understanding the organizational architecture of the brain. Research over the past several decades has shown that the brain is organized in hierarchical networks that shape behavior and cognition, both in healthy and pathological states (Zhou et al., 2006; Honey et al., 2007; Hagmann et al., 2008; Bullmore and Sporns, 2009). At a macroscale level, intrinsic connectivity of parcellated cortical brain regions has been used to characterize neural networks in an attempt to understand their influence on information processing. By examining fluctuations in neural activity, measured by vascular hemodyamic changes, resting-state functional magnetic resonance imaging (rsfMRI) has reliably been used to investigate network connectivity. Networks are characterized as a collection of nodes and

Received Dec. 11, 2017; revised Feb. 12, 2018; accepted Feb. 13, 2018. I thank Drs. Adrian Crawley and Angela Colantonio for review and feedback pertaining to this manuscript.

The author declares no competing financial interests.

Correspondence should be addressed to Reema Shafi, Rehabilitation Sciences Institute, University of Toronto, 500 University Avenue, Suite 160, University of Toronto, Toronto, Ontario M5G 1V7, Canada. E-mail: reema.shafi@mail.utoronto.ca.

DOI:10.1523/JNEUROSCI.3503-17.2018

Copyright $\odot 2018$ the authors $\quad 0270-6474 / 18 / 383154-03 \$ 15.00 / 0$ edges: nodes represented by focal neural parcellations identifiable with three coordinates $(x, y$, and $z)$ and edges as the connections between these nodes (Sporns, 2011). Networks are classified as structural (physical anatomical connections), functional (statistical dependencies among neurophysiological measurements), and effective (one region exerting a causal influence over another) (Friston et al., 2011). Investigation of effective connectivity is a relatively recent development. Corticocortical evoked potential (CCEP) research, which combines invasive (electrode-based EEG recording etc.) and noninvasive (rs-fMRI, diffusion tensor imaging, etc.) methodologies, provides a distinct advantage in such studies by allowing researchers to observe whether stimulation of one site can activate another and drive network variability (Matsumoto et al., 2012).

In a recent article, Shine et al. (2017) combined intracranial stimulation and recording with rs-fMRI to provide valuable insight into the temporal pattern of effective connectivity between three largescale brain networks: the default-mode network (DMN), the salience network (SN), and the frontoparietal network (FPN). These three networks have received considerable attention due to their large spatial extent, which provides a common framework to understand dysfunction across multiple disorders, especially re- lated to cognition (Menon, 2011). Additionally, their dynamic synchronization between resting and attentive states, and ease of visibility, offer a unique opportunity to study complex network dynamics at rest.

Shine et al. (2017) recruited 7 subjects that had depth electrodes implanted for a clinical epilepsy study. Each intracranical electrode was individually assigned membership to a predefined network using rs-fMRI. A pair of electrodes within the nodes of predefined networks were stimulated while evoked responses were recorded from each other pair of electrodes, for a duration of $200 \mathrm{~ms}$. The authors found marked heterogeneity in the networkrelated temporal patterns across subjects; therefore, they used a data-driven approach to identify three spatially and temporally distinct clusters of evoked activity patterns across the 7 subjects. The unsupervised nature of the latter approach adds strength to the study because it does not depend on any a priori hypothesis for expected results, yet the identified clusters aligned with predefined network topology.

Shine et al. (2017) provide evidence that signal propagation among predefined networks occurs along distinct temporal scales. Understanding the temporal modulation of a network can provide unique perspectives on how information is 
transmitted and influenced. The most significant network pattern within the first cluster (which occurred between 11 and $69 \mathrm{~ms}$ after stimulation) was evoked within the SN when electrodes within the FPN and SN were stimulated. The second cluster (82-125 ms) was generated in the FPN as a result of stimulating electrodes within the SN and DMN. The third cluster (132-200 ms) was generated by stimulating electrodes in the DMN, which lead to a more general activation across all three networks: the FPN, SN, and DMN. The authors' post hoc analysis revealed that stimulation within the FPN and SN led to maximal activations in the early window after stimulus, whereas the DMN generated maximal activation in the delayed window after stimulus. The authors also replicated a finding from Keller et al. (2014) demonstrating that intranetwork stimulation generated higher amplitude activation (within the DMN and FPN but not SN) compared with internetwork stimulation. Temporal network connectivity at the individual subject level showed similar activation patterns (i.e., early FPN/SN and late $\mathrm{DMN}$ responses).

Based on the Shine et al. (2017) study, three temporal characteristics may be interpreted to the SN and FPN interaction. First, the fast-evoked activation of the SN (Shine et al., 2017, their Fig. 3) is consistent with this network's key roles (i.e., monitoring and detecting salient events and subsequent access to attention and memory resources) (Menon, 2011). This efficient SN modulation may be supported by a unique regional advantage. The von Economo neurons in the frontoinsular and cingulate cortex (Dijkstra et al., 2018) are rapidly conducting projection neurons. It is suggested that these neurons support sophisticated socialemotional-autonomic functions (Seeley, 2008). Hence, one may conclude that the fast $\mathrm{SN}$ activation is in part supported by its distinct cytoarchitecture, which has evolved to facilitate an efficient, "knee-jerk" assessment of changing (external and internal) environmental cues (Menon, 2011). Second, the SN appears to be tightly coupled with other networks: the DMN, but more so the FPN. This is evidenced by its prevalence across all three temporal clusters. This adaptive coupling reinforces the proposed active role of $\mathrm{SN}$ in network switching (Menon, 2011) and mediating internetwork connectivity (Sridharan et al., 2008). Third, FPN stimulation leads to SN activation in the early temporal cluster, suggesting that FPN depends on the SN for information acquisition. FPN has been associated with initiating and adjusting cognitive control based on salience information (Dosenbach et al., 2008).

Shine et al. (2017) report delayed activation of the DMN (i.e., evoked responses in latter clusters). The authors suggest that this indicates a late influence of the DMN on the SN and FPN (i.e., after these networks have completed early/local processing). They also suggest that the delay in DMN influence may expedite the integration of information processing, albeit over time. While the authors attribute this delayed DMN activation to multisynaptic processing, and imply its role as an "influencer" acting after early-level processing ( $\mathrm{SN}$ and FPN), the interpretation of delayed DMN activation appears to be more challenging. Shine et al. (2017) report that most of their DMN electrodes were located in the medial temporal lobe regions for clinical reasons. Here the polysynaptic hypothesis, as suggested by Shine et al. (2017), is well supported, as the medial temporal lobe is a convergent zone of cortical processing and receives input from all sensory modalities creating multiple subsystems (Simons and Spiers, 2003; Squire et al., 2004). This may create a bottleneck effect contributing to longer latencies. Nevertheless, there are other explanations that warrant consideration. For example, there is evidence of reduced DMN recruitment and alterations in hippocampal networks associated with epilepsy, which may also have contributed to the delayed DMN activation (James et al., 2013). Another study-specific limitation, clearly unavoidable, is the somewhat rostral placement of stimulation electrodes, which may have biased recordings toward delayed activation. Furthermore, it has been suggested that the DMN plays a role in functional integration of information across segregated brain regions (Hagmann et al., 2008). This proposed role could explain the delayed, and more importantly, sustained activation. Can the delayed yet sustained processing within the DMN be the brain's intrinsic effort to reorganize and bind information in a relational pattern to facilitate later retrieval? The absence of DMN activation in the early clusters, its large spatial span across the cortex, and its estimated $20 \%$ consumption of neural resources may hint toward a global, more robust role in information processing. However, this remains to be explored.

A few considerations suggest that the findings of Shine et al. (2017) should be interpreted with caution. One of the most evident limitations of interpreting CCEP data from epileptic participants is the lack of generalizability to control populations (Kunieda et al., 2015). Although the stimulations do not trigger a recordable epileptic discharge, there may be unexplored alterations in brain activity with little to no consequences for behavior. Also, while it has been hypothesized that intracranial electrode stimulation elicits activation of the deep (layer IV-VI) cortical layers (Keller et al., 2014), the lack of such validation reduces interpretability and may require methodological clarification. Despite these limitations, CCEP studies offer an unprecedented advantage to advance the understanding of neural organization patterns, albeit in a pathological state.

To reiterate, the results from Shine et al. (2017) support the notion that, despite obvious heterogeneity within the connectome, the macroscale interactions within and between neural networks follow a temporal hierarchy. Their evidence lends further support to the conceptual framework that the region's architectural thresholds may help optimize signal propagation and foster an environment of efficient connectivity. Understanding this more global temporal hierarchy along with regional cytoarchitectural characteristics may provide insights about the connectome (van den Heuvel et al., 2015). CCEP is a powerful tool that can identify the timevarying "chronnectome" (Calhoun et al., 2014) and may possibly help predict behavior by increasing our understanding of the hierarchical organization underlying efficient information processing, and alterations that may manifest in pathological disease states.

\section{References}

Bullmore E, Sporns O (2009) Complex brain networks: graph theoretical analysis of structural and functional systems. Nat Rev Neurosci 10:186-198. CrossRef Medline

Calhoun VD, Miller R, Pearlson G, Adalı T (2014) The chronnectome: time-varying connectivity networks as the next frontier in fMRI data discovery. Neuron 84:262-274. CrossRef Medline

Dijkstra AA, Lin LC, Nana AL, Gaus SE, Seeley WW (2018) von Economo neurons and fork cells: a neurochemical signature linked to monoaminergic function. Cereb Cortex 28: 131-144. CrossRef Medline

Dosenbach NU, Fair DA, Cohen AL, Schlaggar BL, Petersen SE (2008) A dual-networks architecture of top-down control. Trends Cogn Sci 12:99-105. CrossRef Medline

Friston KJ (2011) Functional and effective connectivity: a review. Brain Connect 1:13-36. CrossRef Medline 
Hagmann P, Cammoun L, Gigandet X, Meuli R, Honey CJ, Wedeen VJ, Sporns O (2008) Mapping the structural core of human cerebral cortex. PLoS Biol 6:e159. CrossRef Medline

Honey CJ, Kötter R, Breakspear M, Sporns O (2007) Network structure of cerebral cortex shapes functional connectivity on multiple time scales. Proc Natl Acad Sci U S A 104: 10240-10245. CrossRef Medline

James GA, Tripathi SP, Ojemann JG, Gross RE, Drane DL (2013) Diminished default mode network recruitment of the hippocampus and parahippocampus in temporal lobe epilepsy. J Neurosurg 119:288-300. CrossRef Medline

Keller CJ, Honey CJ, Megevand P, Entz L, Ulbert I, Mehta AD (2014) Mapping human brain networks with cortico-cortical evoked potentials. Philos Trans R Soc Lond B Biol Sci 369: 1653. CrossRef Medline

Kunieda T, Yamao Y, Kikuchi T, Matsumoto R (2015) New approach for exploring cerebral functional connectivity: review of corticocortical evoked potential. Neurol Med Chir (Tokyo) 55:374-382. CrossRef Medline
Matsumoto R, Nair DR, Ikeda A, Fumuro T, Lapresto E, Mikuni N, Bingaman W, Miyamoto S, Fukuyama H, Takahashi R, Najm I, Shibasaki H, Lüders HO (2012) Parietofrontal network in humans studied by corticocortical evoked potential. Hum Brain Mapp 33:2856-2872. CrossRef Medline

Menon V (2011) Large-scale brain networks and psychopathology: a unifying triple network model. Trends Cogn Sci 15:483-506. CrossRef Medline

Mesulam MM (1990) Large-scale neurocognitive networks and distributed processing for attention, language, and memory. Ann Neurol 28:597-613. CrossRef Medline

Seeley WW (2008) Selective functional, regional, and neuronal vulnerability in frontotemporal dementia. Curr Opin Neurol 21: 701-707. CrossRef Medline

Shine JM, Kucyi A, Foster BL, Bickel S, Wang D, Liu H, Poldrack RA, Hsieh LT, Hsiang JC, Parvizi J (2017) Distinct patterns of temporal and directional connectivity among intrinsic networks in the human brain. J Neurosci 37: 9667-9674. CrossRef Medline
Simons JS, Spiers HJ (2003) Prefrontal and medial temporal lobe interactions in long-term memory. Nat Rev Neurosci 4:637-648. CrossRef Medline

Sporns O (2011). Networks of the Brain. Cambridge, UK:MIT Press.

Squire LR, Stark CE, Clark RE (2004) The medial temporal lobe. Annu Rev Neurosci 27: 279-306. CrossRef Medline

Sridharan D, Levitin DJ, Menon V (2008) A critical role for the right fronto-insular cortex in switching between central-executive and defaultmode networks. Proc Natl Acad Sci U S A 105: 12569-12574. CrossRef Medline

van den Heuvel MP, Scholtens LH, Feldman Barrett L, Hilgetag CC, de Reus MA (2015) Bridging cytoarchitectonics and connectomics in human cereb cortex. J Neurosci 35:1394313948. CrossRef Medline

Zhou C, Zemanová L, Zamora G, Hilgetag CC, Kurths J (2006) Hierarchical organization unveiled by functional connectivity in complex brain networks. Phys Rev Lett 97:238103. CrossRef Medline 